\title{
Características da madeira de Cunninghamia lanceolata (Chinese fir)
}

\section{Characteristics of Cunninghamia lanceolata (Chinese fir) wood}

\author{
Magnos Alan Vivian ${ }^{1}$ (D), Alexia Maria de Oliveira da Silva ${ }^{1}$ (D), Karina Soares Modes $^{1}$ (D), \\ Mário Dobner Júnior ${ }^{1}$ (D) Francides Gomes da Silva Júnior ${ }^{2}$ (1) \\ ${ }^{1}$ Universidade Federal de Santa Catarina - UFSC, Curitibanos, SC, Brasil \\ ${ }^{2}$ Escola Superior de Agricultura "Luiz de Queiroz" - ESALQ, Universidade de São Paulo - USP, Piracicaba, SP, Brasil
}

Como citar: Vivian, M. A., Silva, A. M. O., Modes, K. S., Dobner Júnior, M., Silva Júnior, F. G. (2021). Características da madeira de Cunninghamia lanceolata (Chinese fir). Scientia Forestalis, 49(131), e3581.

https://doi.org/10.18671/scifor.v49n131.01

\begin{abstract}
Resumo
O presente estudo teve por objetivo avaliar as características físico-químicas e anatômicas da madeira de Cunninghamia lanceolata, aos 24 anos de idade, proveniente de plantação experimental realizada no estado de Santa Catarina. Foram colhidas árvores médias do povoamento, das quais retiraram-se discos em diferentes alturas do fuste para avaliação da densidade básica, composição química e anatômica (morfologia dos traqueídeos e indicadores anatômicos de qualidade para produção de papel). Os resultados obtidos permitiram classificar a madeira de $C$. lanceolata como leve ou de baixa densidade $\left(0,350 \mathrm{~g} \mathrm{~cm}^{-3}\right)$. Em relação à composição química, a mesma apresentou teores de cinzas $(0,35 \%)$ e extrativos $(4,36 \%)$ dentro da faixa normalmente observada para coníferas, porém valores elevados de lignina (33,25\%) e baixo de holocelulose (62,39\%). Os traqueídeos são largos (40,55 $\mu \mathrm{m})$, de comprimento moderadamente longo $(2,14 \mathrm{~mm})$, de parede espessa $(3,51 \mu \mathrm{m})$, que variam de forma significativa no sentido radial (medula-casca). Os traqueídeos apresentam excelentes indicadores anatômicos para produção de papel, como índice de Runkel $(0,23)$, coeficiente de flexibilidade $(81,94 \%)$ e fração parede $(18,06 \%)$. Conclui-se, com base nas características da madeira, que a C. lanceolata apresenta bom potencial para uso no Brasil, podendo ser uma alternativa viável para suprir a demanda de matéria-prima do setor industrial, especialmente para o segmento de celulose e papel.
\end{abstract}

Palavras-chave: Qualidade da madeira; Conífera; Pinheiro-chinês; Espécie alternativa.

\begin{abstract}
The present study aimed to evaluate the physical, chemical and anatomical characteristics of Cunninghamia lanceolata wood, at 24 years of age, from an experimental plantation carried out in Santa Catarina state. Average plantation trees were harvested, disks at different stem heights were removed to evaluate the basic density, chemical and anatomical composition (tracheid morphology and anatomical quality indicators for paper production). The results obtained made it possible to classify the $C$. lanceolata wood as of slight or low density $\left(0.350 \mathrm{~g} \mathrm{~cm}^{-3}\right)$. Regarding the chemical composition, it presented ash $(0.35 \%)$ and extractives $(4.36 \%)$ contents within the range normally observed for conifers, but had high values of lignin (33.25\%) and low ones of holocellulose (62.39\%). The tracheids are wide $(40.55 \mu \mathrm{m})$, moderately long in length $(2.14 \mathrm{~mm})$, thick walled $(3.51 \mu \mathrm{m})$, with significant variation in the radial direction (pith-bark). Tracheids are excellent anatomical quality indicators for paper production, such as Runkel index (0.23), flexibility coefficient (81.94\%) and wall fraction (18.06\%). Based on the wood characteristics it is concluded that C. lanceolata has a good potential for use in Brazil. It may be a viable alternative to supply the demand for raw material from the industrial sector, especially for the pulp and paper segment.
\end{abstract}

Keywords: Wood quality; Conifers; Chinese fir; Alternative species.

Fonte de financiamento: Nenhuma.

Conflito de interesse: Nada a declarar.

Autor correspondente: magnos.alan@ufsc.br

Recebido: 21 julho 2020.

Aceito: 2 outubro 2020

Editor: Paulo Henrique Müller Silva.

(c) (i) Este é um artigo publicado em acesso aberto (Open Access) sob a licença Creative Commons Attribution, que permite uso, distribuição e reprodução em qualquer meio, sem restrições desde que o trabalho original seja corretamente citado. 


\section{INTRODUÇÃO}

O Brasil é um país que apresenta alto potencial florestal, seja para o cultivo de folhosas ou de coníferas, com condições edafoclimáticas que possibilitam o plantio de uma gama de espécies nativas ou exóticas. Atualmente, os dois principais gêneros plantados no país são o Eucalyptus e Pinus, que fornecem matéria-prima para o setor industrial. Porém a busca por novas espécies que possam fornecer madeira de qualidade é constante, ainda mais em um país que possui dimensões continentais e condições edafoclimáticas que possibilitam o cultivo de uma infinidade de espécies florestais do mundo, ampliando assim o leque de opções de espécies para os mais diversos usos.

Entre as espécies que merecem atenção está Cunninghamia lanceolata (Lamb.) Hook., conhecida como "pinheiro-chinês", ou internacionalmente como "Chinese fir". Esta é uma das espécies mais importantes e plantadas na China, ocupando uma área de aproximadamente 25\% das plantações florestais do sul do país (Zheng et al., 2015; Wen et al., 2018).

C. lanceolata é uma conífera pertencente à família Cupressaceae, que ocorre naturalmente nas regiões central e sul da China, em altitudes que variam entre 600 a 1.300 m, em locais onde o clima se caracteriza como temperado e subtropical úmido, com verões quentes e chuvosos, bem como invernos frios e secos, entretanto sem déficit hídrico (Empresa Brasileira de Pesquisa Agropecuária, 1988).

A madeira da espécie é considerada de ótima qualidade, sendo amplamente utilizada na China para construção de edifícios, casas, pontes, navios, móveis, bem como demonstra potencial para fabricação de polpa celulósica (Minghe \& Ritchie, 1999; Chen et al., 2017; Li et al., 2019). Além disso, a mesma apresenta madeira de alta durabilidade natural (Fung, 1993; Wang et al., 2011), e extrativos com potencial farmacológico (Xin et al., 2012).

Em relação ao seu crescimento nas condições edafoclimáticas brasileiras, pesquisadores relatam incrementos médios anuais (IMA) variando entre 28 e $36 \mathrm{~m}^{3} \mathrm{ha}^{-1} \mathrm{ano}^{-1}$, confirmando seu potencial de cultivo, especialmente para o planalto sul do país (Empresa Brasileira de Pesquisa Agropecuária, 1988; Dobner Júnior et al., 2017). Apesar de apresentar bom crescimento e qualidade, como já relatado anteriormente, existem poucos estudos no Brasil avaliando as características tecnológicas da madeira de C. lanceolata.

Desta forma, para o melhor aproveitamento da madeira é essencial à avaliação e conhecimento de suas propriedades físico-químicas e anatômicas, que indicarão suas aptidões, com destaque para produção de polpa celulósica e papel. Entre estas propriedades se destacam a densidade básica, a composição química e as dimensões dos traqueídeos, que afetam diretamente os parâmetros de polpação e propriedades do papel produzido.

Assim, o objetivo deste estudo foi avaliar as características físico-químicas e anatômicas da madeira de C. lanceolata, aos 24 anos de idade, proveniente de uma plantação experimental do estado de Santa Catarina.

\section{MATERIAL E MÉTODOS}

O estudo foi conduzido utilizando-se a madeira de Cunninghamia lanceolata, com 24 anos de idade, coletada em plantação experimental da empresa Florestal Gateados, localizada na cidade de Campo Belo do Sul/SC (latitude $27^{\circ} 57^{\prime} 06^{\prime \prime}$, Longitude $50^{\circ} 49^{\prime} 08^{\prime \prime} \mathrm{O}$ e $920 \mathrm{~m}$ de altitude) (Figura 1). A plantação de espaçamento inicial de $2,5 \times 2,5 \mathrm{~m}$ (1.600 árv/ha), foi submetida ao trato silvicultural de poda até à altura de 5,8 $\mathrm{m}$, porém não tinha recebido desbaste até a data da coleta. O clima da região, de acordo com a classificação de Köppen, é $\mathrm{Cfb}$, mesotérmico, subtropical úmido, sem estações secas definidas e com ocorrência de geadas severas (Dobner Júnior et al., 2017). 

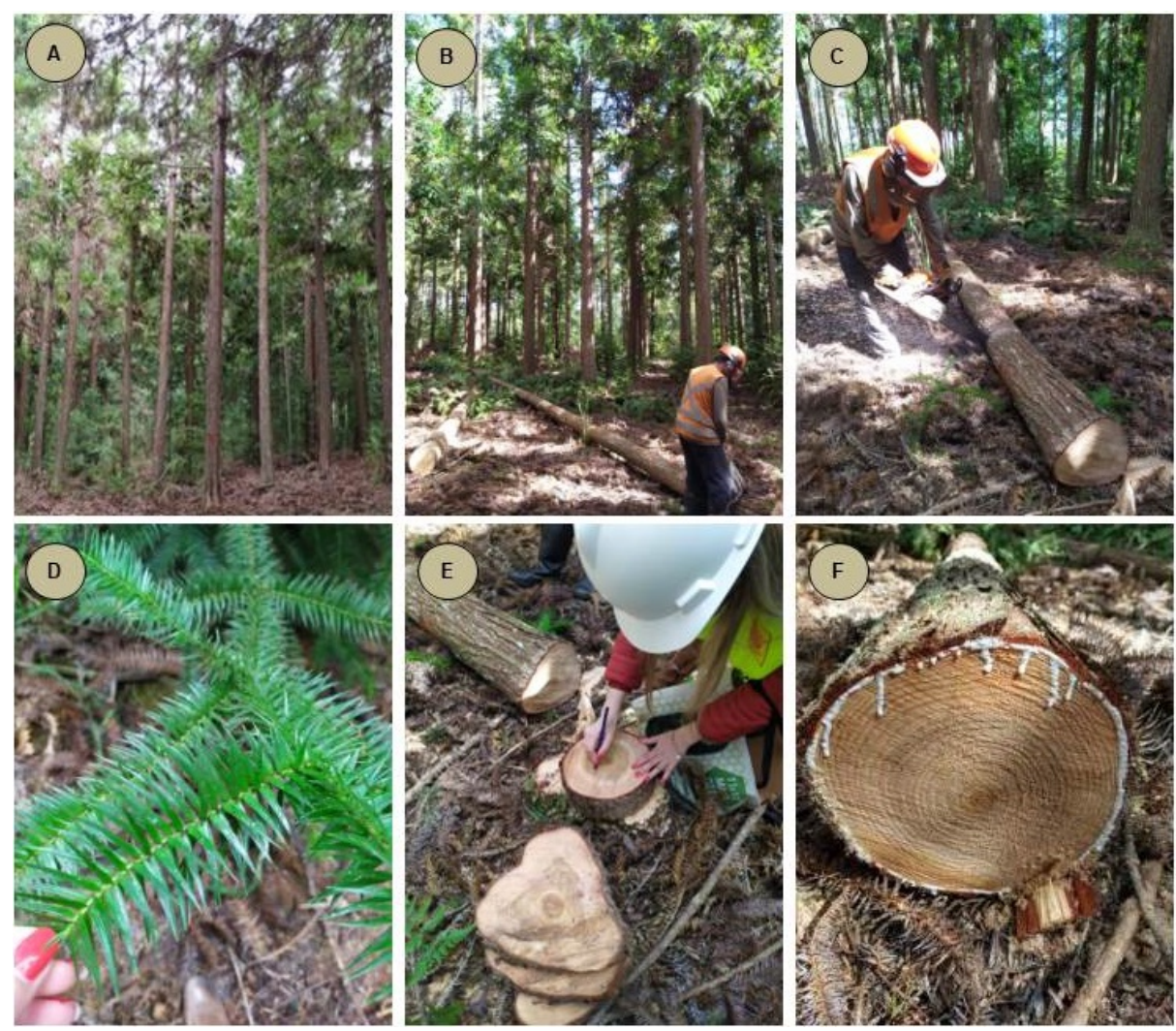

Figura 1. Coleta da madeira de C. lanceolata. (A) Aspecto da plantação; (B) Derrubada das árvores; (C) Seccionamento nas posições de amostragem; (D) Aspecto das folhas; (E) Marcação das amostras; (F) Exsudação de extrativos na transição madeira-casca.

Selecionaram-se aleatoriamente três árvores médias de um povoamento de 0,2 ha (valores médios obtidos: $23,7 \mathrm{~cm}$ de diâmetro à altura do peito (DAP=1,30 $\mathrm{m}$ do solo), 19,6 m de altura total e 15,5 $\mathrm{m}$ de altura comercial, 0,6 cm de espessura de casca), evitando-se as árvores próximas às bordaduras, das quais retiraram-se discos (com $3,0 \mathrm{~cm}$ de espessura) nas posições: 0 (base), DAP, 25, 50, 75 e 100\% da altura comercial (diâmetro de ponta fina de 8 $\mathrm{cm}$ ) para determinação da densidade básica e das propriedades químicas e anatômicas.

\section{Densidade básica}

A densidade básica da madeira, e sua variação longitudinal no fuste, foi determinada a partir dos discos retirados ao longo da altura comercial, que foram seccionados em duas cunhas livres de defeitos (nós e lenhos de reação) e, em seguida, imersas em água até à saturação completa. Na sequência determinou-se a massa seca e o volume saturado de cada uma das cunhas, de acordo com as recomendações da Norma Brasileira Regulamentadora 11.941 (Associação Brasileira de Normas Técnicas - ABNT, 2003).

Com base nos valores de densidade em cada posição/altura do fuste obtido pela média das duas cunhas, calculou-se a densidade básica ponderada, utilizando os diâmetros dos discos nas diferentes alturas e suas respectivas densidades básicas, conforme a Equação 1 (citada por Segura, 2015).

$D b p=\frac{1}{2} \frac{\left(D_{B}^{2}+D_{d a p}^{2}\right) \cdot\left(d_{B}+d_{d a p}\right)+\left(D_{d a p}^{2}+D_{25 \%}^{2}\right) \cdot\left(d_{d a p}+d_{25 \%}\right)+\left(D_{25 \%}^{2}+D_{50 \%}^{2}\right) \cdot\left(d_{25 \%}+d_{50 \%}\right)+\left(D_{50 \%}^{2}+D_{75 \%}^{2}\right) \cdot\left(d_{50 \%}+d_{75 \%}\right)+\left(D_{75 \%}^{2}+D_{100 \%}^{2}\right) \cdot\left(d_{75 \%}+d_{100 \%}\right)}{D_{B}^{2}+D_{100 \%}^{2}+2 \cdot\left(D_{d a p}^{2}+D_{25 \%}^{2}+D_{50 \%}^{2}+D_{55 \%}^{2}\right)}$

Em que: $\mathrm{Dbp}=$ densidade básica ponderada $\left(\mathrm{g} \mathrm{cm}^{-3}\right)^{3} ; \mathrm{D}_{\mathrm{B}}, \mathrm{D}_{\mathrm{dap}}, \mathrm{D}_{25 \%}, \mathrm{D}_{50 \%}, \mathrm{D}_{75 \%}$ e $\mathrm{D}_{100 \%}=$ diâmetros dos discos em diferentes alturas ( $\mathrm{cm}$ ); $d_{B}, d_{d a p}, d_{25 \%}, d_{50 \%}, d_{75 \%}$ e $d_{100 \%}=$ densidades básicas em diferentes alturas $\left(\mathrm{g} \mathrm{cm}^{-3}\right)$. 


\section{Propriedades químicas}

Determinou-se a composição química da madeira por meio de uma amostra composta, com os discos retirados ao longo do fuste. Esta foi transformada em serragem utilizando-se um moinho do tipo Willey, que foi classificada em peneiras vibratórias (40 e 60 mesh), selecionando-se a fração que ficou retida na peneira de 60 mesh.

Foi determinado, em triplicata, os seguintes compostos: cinzas (Technical Association of Pulp and Paper Industry, 2002), extrativos totais (Technical Association of Pulp and Paper Industry, 1997), lignina e holocelulose (metodologia descrita por Vivian et al., 2015).

\section{Propriedades anatômicas}

Para a análise das características anatômicas da madeira de $C$. lanceolata foram retiradas seções radiais dos discos da base com $2 \mathrm{~cm}$ de largura, no sentido medula-casca (sempre no maior raio), e destas marcaram-se as porções de 0, 25, 50, 75 e 100\% ( 0 = próximo a medula; $100 \%$ = próximo a casca). Essas porções foram transformadas em pequenos fragmentos (palitos) e submetidos ao processo de maceração, em tubos de ensaio contendo ácido acético, ácido nítrico e água (proporção de 5:2:1) para realizar a separação/individualização dos traqueídeos. Os tubos de ensaio foram mantidos em banho-maria na temperatura próxima de $100^{\circ} \mathrm{C}$ por um período de aproximadamente $1 \mathrm{~h}$, conforme a metodologia proposta por Barrichelo \& Foelkel (1983). Finalizado este procedimento, o material foi lavado com água destilada até remoção completa das substâncias utilizadas no processo.

A partir do material macerado foram montadas lâminas com adição de 1 gota de safranina (1\%), glicerina e água, visando a obtenção de imagens dos traqueídeos, a partir de uma lupa (aumento de 6,3 vezes para o comprimento) e um microscópio (aumento de 400 vezes para largura e diâmetro do lúmen). Foram mensuradas as seguintes dimensões: comprimento, largura e diâmetro de lúmen, seguindo a norma da IAWA (International Association of Wood Anatomists, 1989). Após a obtenção destas medidas, calcularam-se: espessura da parede celular, fração parede, coeficiente de flexibilidade, índice de enfeltramento e índice de Runkel, com base nas Equações 2, 3, 4, 5 e 6, respectivamente. Foram avaliados 35 traqueídeos por posição ( 35 traqueídeos $\times 5$ posições $\times 3$ árvores $=525$ traqueídeos).

$$
\begin{aligned}
& E=\left(\frac{L-D L}{2}\right) \\
& F P=\left(\frac{2 \cdot E}{L}\right) \cdot 100 \\
& C F=\left(\frac{D L}{L}\right) \cdot 100 \\
& I E=\left(\frac{C}{\frac{L}{1000}}\right) \\
& I R=\left(\frac{2 \cdot E}{D L}\right)
\end{aligned}
$$

Em que: $E=$ espessura da parede celular $(\mu \mathrm{m}) ; \mathrm{L}=$ largura do traqueídeo $(\mu \mathrm{m}) ; \mathrm{DL}=$ diâmetro do lúmen $(\mu \mathrm{m}) ; \mathrm{FP}=$ fração parede $(\%) ; \mathrm{CF}=$ coeficiente de flexibilidade $(\%) ; \mathrm{IE}=$ índice de enfeltramento; $C$ = comprimento do traqueídeo $(\mathrm{mm}) ; \mathrm{IR}=$ índice de Runkel. 


\section{Análise dos dados}

Os dados obtidos foram armazenados e analisados com o auxílio de planilhas eletrônicas e software estatístico Sisvar (versão 5.7). Para densidade básica e morfologia dos traqueídeos realizou-se a análise de variância (ANOVA) e quando significativo foi aplicado o teste de Tukey, ao nível de $5 \%$ de probabilidade, nos valores axiais (densidade) ou sentido radial (morfologia) da madeira. Para a composição química foi realizada a análise descritiva dos compostos, indicando o desvio padrão dos dados.

\section{RESULTADOS E DISCUSSÃO}

\section{Densidade básica}

A densidade básica, média e ponderada, da madeira de $C$. lanceolata aos 24 anos, bem como sua variação longitudinal ao longo do fuste comercial, pode ser observada na Figura 2. Com base na classificação proposta pela International Association of Wood Anatomists (1989), a densidade básica da madeira de $C$. lanceolata é considerada leve ou de baixa densidade $\left(<0,40 \mathrm{~g} \mathrm{~cm}^{-3}\right)$.
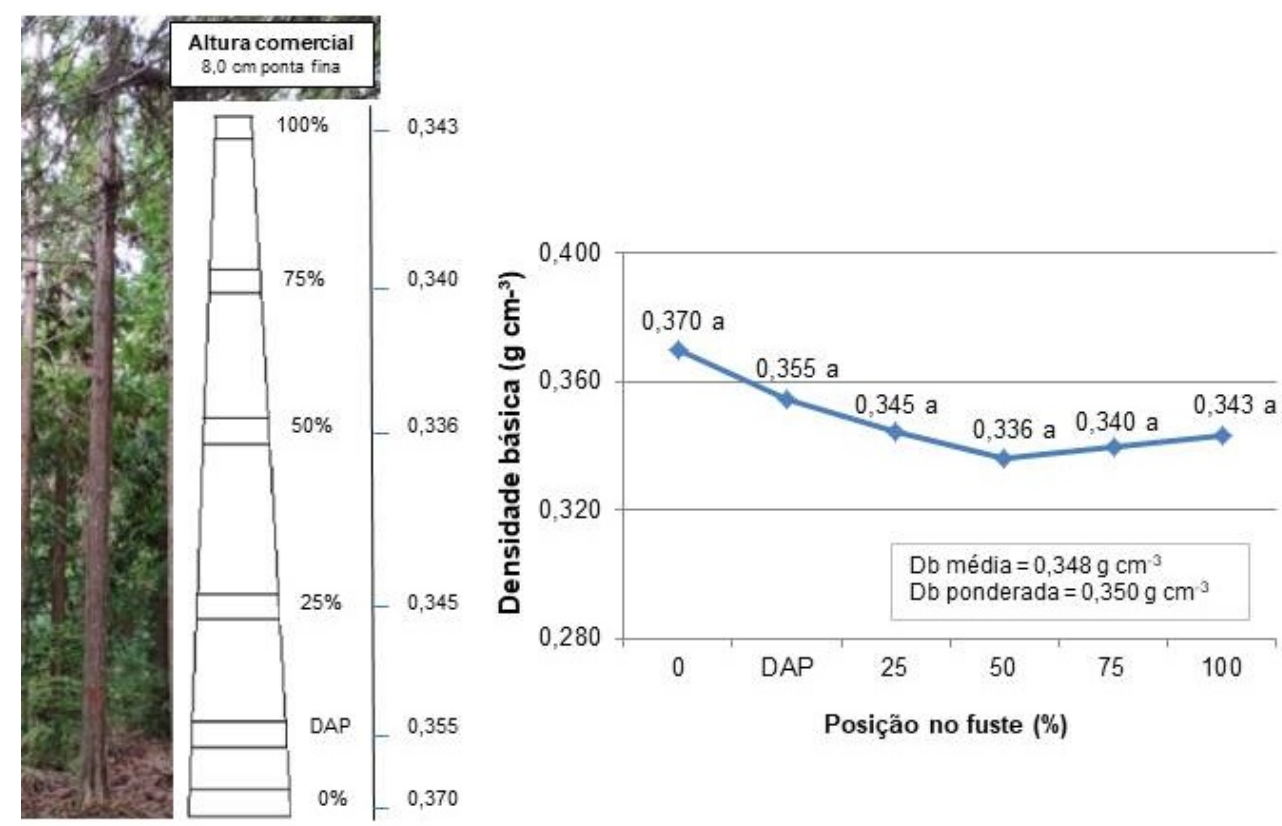

Figura 2. Densidade básica da madeira de C. lanceolata aos 24 anos de idade, plantada em Campo Belo do Sul/SC. Média seguidas da mesma letra não diferem entre si pelos teste de Tukey.

Não foi observada diferença estatística entre os valores de densidade básica avaliados ao longo do fuste da espécie, contudo verifica-se uma tendência de decréscimo dessa propriedade da base em direção ao topo com uma amplitude de 7,30\% entre as médias registradas nos extremos. Esse comportamento é comum em espécies de coníferas, conforme observado por Mattos et al. (2011) e Melo et al. (2013).

A média de densidade básica obtida para a madeira de $C$. lanceolata no presente estudo situase dentro da faixa de 0,229 a $0,510 \mathrm{~g} \mathrm{~cm}^{-3}$ citada por Duan et al. (2016), que avaliaram clones da mesma espécie, aos 10 anos de idade, oriundos de seis províncias da China. A densidade básica da madeira da espécie situa-se próxima à encontrada por Fung (1993), que avaliou árvores com idade similar (25 anos), porém foi superior ao valor encontrado por Zheng et al. (2015), que trabalharam com árvores mais jovens (12 anos), com metade da idade das utilizadas neste estudo.

Na Tabela 1 observa-se a comparação da densidade básica da madeira de C. lanceolata com outras espécies de coníferas, como o Cupressus lusitanica, Cryptomeria japonica, Pinus taeda, Pinus elliottii e Pinus sylvestris. 
Tabela 1. Comparação da densidade básica da madeira de C. lanceolata aos 24 anos de idade, plantada em Campo Belo do Sul/SC, com outras espécies de coníferas.

\begin{tabular}{cccc}
\hline Espécie & Idade (anos) & Densidade básica $\mathbf{( \mathbf { ~ c m } ^ { - 3 } )}$ & Fonte \\
\hline C. lanceolata & 24 & 0,350 & Presente estudo \\
C. lanceolata & 12 & 0,275 & Zheng et al. (2015) \\
C. lanceolata & 20 & 0,332 & Guo et al. (2018) \\
C. Ianceolata & 25 & 0,315 & Fung (1993) \\
C. japonica & 35 & 0,356 & Trianoski et al. (2013a) \\
C. Iusitanica & 16 & 0,375 & Vivian et al. (2020) \\
C. Iusitanica & 19 & 0,396 & Almeida et al. (2017) \\
P. taeda & 17 & 0,415 & Trianoski et al. (2013b) \\
P. taeda & 21 & 0,435 & Vivian et al. (2015) \\
P. elliottii & 15 & 0,472 & Palermo et al. (2004) \\
P. sylvestris & 45 & 0,436 & Vivian et al. (2015) \\
\hline
\end{tabular}

Além disso, como observado na Tabela 1, a densidade básica da madeira de $C$. lanceolata se assemelha aos valores citados para as espécies $C$. lusitanica e $C$. japonica, entretanto é inferior quando comparada as principais espécies de Pinus utilizadas no setor florestal brasileiro. Atualmente o $P$. taeda é a espécie mais utilizada no país para fabricação de polpa celulósica de "fibra longa", com as fábricas totalmente adaptadas às características da madeira desta (Vivian et al., 2015).

Muito se discute na literatura sobre a faixa de densidade ideal das madeiras para fabricação de celulose $\left(0,40\right.$ a 0,55 $\left.\mathrm{g} \mathrm{cm}^{-3}\right)$ (Dias \& Simonelli, 2013), porém segundo Fantuzzi Neto (2012), madeiras de baixa e alta densidade podem proporcionar os mesmos rendimentos, desde que se tenha atenção com os parâmetros de polpação utilizados, como fator $\mathrm{H}$ (é uma única variável utilizada para expressar tempo e temperatura do cozimento). A madeira de $C$. lanceolata apresenta densidade abaixo da faixa mencionada, porém este aspecto pode apresentar algumas vantagens, como mencionado por Vivian et al. (2020), que citam que madeiras de baixa densidade são mais fáceis de picar, impregnar e cozinhar, porém com rendimento menor. Por fim, outros aspectos devem ser levados em consideração, e que estão intimamente relacionados com a densidade, sendo afetados pela mesma, como consumo específico de madeira, carga de reagentes, geração de rejeitos, custos de transporte, entre outros.

\section{Propriedades químicas}

Na Figura 3 podem ser observados os valores médios da composição química da madeira de C. Ianceolata. Os valores obtidos para cinzas $(0,35 \%)$ e extrativos $(4,36 \%)$ situam-se na faixa de amplitude citada por Klock \& Andrade (2013) para coníferas (cinzas: 0,2 e 0,5\%; extrativos: $2,0$ e $8,0 \%)$. Com base nas faixas citadas pelos mesmos autores para holocelulose $(65,0 \mathrm{e}$ $73,0 \%$ ) e lignina (26,0 e 30,0\%), o teor de holocelulose observado (62,39\%) está abaixo do esperado e o teor de lignina $(33,25 \%)$ acima .

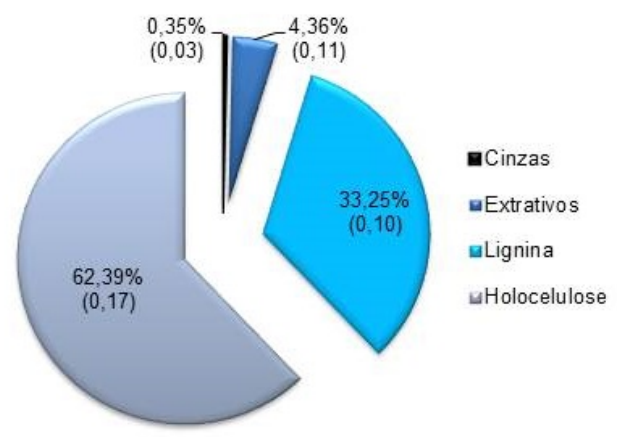

Figura 3. Composição química da madeira de C. lanceolata aos 24 anos de idade, plantada em Campo Belo do Sul/SC. Desvio padrão entre parênteses. 
Na Tabela 2 observa-se a comparação das características químicas da madeira de C. lanceolata com outras espécies de coníferas. Os valores obtidos no presente estudo estão próximos aos citados por Li et al. (2019), que avaliaram a composição química da madeira de cerne e alburno da C. lanceolata, com valores variando entre 0,15 e 0,31\% para cinzas, entre 1,42 e 5,30\% para extrativos, e 31,18 e 34,16\% para lignina.

Tabela 2. Comparação da composição química da madeira de C. lanceolata aos 24 anos de idade, plantada em Campo Belo do Sul/SC, com outras espécies de coníferas.

\begin{tabular}{lcccccc}
\hline Espécie & $\begin{array}{c}\text { Idade } \\
\text { (anos) }\end{array}$ & TC (\%) & TE (\%) & TL (\%) & HC (\%) & Fonte \\
\hline C. lanceolata & 24 & 0,35 & 4,36 & 33,25 & 62,39 & Presente estudo \\
C. lanceolata & 18 & 0,55 & 9,93 & 31,82 & 57,70 & César et al. (2017) \\
C. lanceolata & 37 & - & - & 33,30 & 67,70 & Chen et al. (2017) \\
C. japonica & 22 & - & 4,90 & 32,70 & 62,50 & Pereira et al. (2003) \\
C. japonica & 35 & 0,39 & 3,77 & 35,46 & - & Fonte et al. (2017) \\
C. lusitanica & 16 & 0,27 & 2,13 & 32,52 & 65,08 & Vivian et al. (2020) \\
C. lusitanica & 19 & 0,51 & 4,08 & 36,21 & 59,19 & Almeida et al. (2016) \\
P. taeda & 16 & 0,36 & 3,65 & 29,83 & 66,17 & Ampessan et al. (2015) \\
P. taeda & 21 & - & 2,83 & 26,71 & 70,46 & Vivian et al. (2015) \\
P. sylvestris & 45 & - & 6,40 & 25,61 & 67,99 & Vivian et al. (2015) \\
\hline
\end{tabular}

Em que: $\mathrm{TC}=$ teor de cinzas; TE = teor de extrativos totais; $\mathrm{TL}=$ teor de lignina; $\mathrm{HC}=$ teor de holocelulose.

Os valores obtidos para teor de cinzas, extrativos, lignina e holocelulose da madeira de C. lanceolata do presente estudo são consoantes com alguns parâmetros observados por outros autores para as espécies C. lusitanica e C. japonica. Já quando comparada com as espécies do gênero Pinus, a madeira de C. lanceolata apresenta maior teor de lignina e menor teor de holocelulose.

O elevado teor de lignina e baixo teor de holocelulose da madeira de C. lanceolata, especialmente em relação ao Pinus, são prejudiciais se destinada para produção de polpa celulósica, pois vai demandar mais reagentes para individualizar os traqueídeos e resultar em menor rendimento, respectivamente. Entretanto, deve-se levar em consideração a questão do melhoramento genético, que no gênero Pinus já esta avançado, e que a C. lanceolata é praticamente "selvagem", desta forma apresentando margem para melhoria de suas propriedades. Essa questão vale tanto para as características da madeira da espécie, quanto para o seu potencial de crescimento e produção florestal.

Outro aspecto importante é o teor de extrativos, que deve ser baixo de preferência, pois estes consomem reagentes e podem gerar incrustações nos equipamentos e na polpa celulósica, conhecidos como pitch (Barbosa et al., 2005). O valor observado para a madeira de C. lanceolata é elevado (4,36\%) quando comparado com $P$. taeda, o que é indesejável para o processo de polpação, porém como já mencionado esta dentro da faixa esperada para coníferas. Já em relação ao teor de cinzas observado $(0,35 \%)$, este pode ser considerado baixo, sendo desejável para o processo de polpação, pois evita problemas como corrosões, entupimentos e incrustações nos equipamentos industriais (Fredo et al., 1999).

\section{Propriedades anatômicas}

Os valores médios obtidos para a morfologia dos traqueídeos e indicadores anatômicos da madeira de C. lanceolata, bem como sua variação no sentido radial, podem ser observados nas Figuras 4 e 5 . Com base na contagem dos anéis de crescimento, que são bem demarcados, observou-se que as posições radiais de amostragem representavam, aproximadamente, os seguintes anos das árvores: $0 \%=2$ anos; $25 \%=8$ anos; $50 \%=14$ anos; $75 \%=18$ anos e $100 \%=24$ anos. 

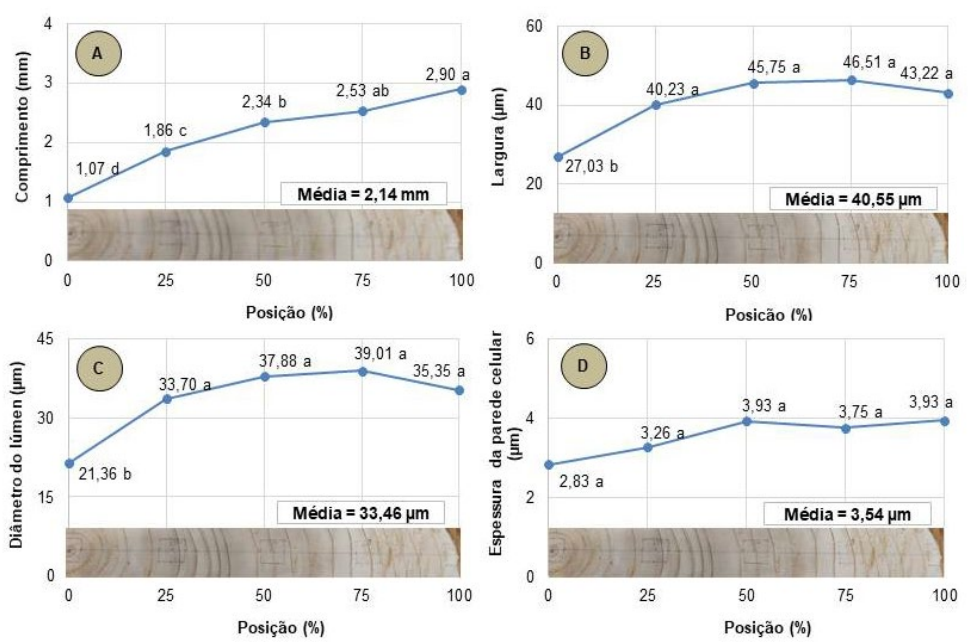

Figura 4. Dimensões dos traqueídeos ao longo do raio da madeira de C. lanceolata aos 24 anos de idade, plantada em Campo Belo do Sul/SC. (A) Comprimento; (B) Largura; (C) Diâmetro do lúmen; (D) Espessura da parede celular. Média seguidas da mesma letra não diferem entre si pelos teste de Tukey.

Analisando a variação das dimensões dos traqueídeos, nota-se que estas diferiram significativamente no sentido radial (medula-casca), com exceção da espessura da parede celular, apresentando valores menores próximos a medula, crescentes no sentido radial, com tendência de estabilização próxima a casca. Não foi possível indicar uma transição clara entre madeira juvenil e adulta, porém analisando conjuntamente a morfologia dos traqueídeos, acredita-se que a mesma ocorra entre as posições 50 e $75 \%$ do raio, que equivalem aproximadamente a 14 e 18 anos. Para o gênero Pinus, por exemplo, estudos demonstram que a transição entre madeira juvenil e adulta ocorre entre 13 e 18 anos de idade (Ballarin \& Palma, 2003; Dobner Júnior et al., 2018).

Com base no comprimento médio dos traqueídeos $(2,14 \mathrm{~mm})$, é possível classificá-los como moderadamente longos (entre 1,60 e 2,20 mm), conforme divisão proposta por Metcalfe \& Chalk (1983). A espessura da parede celular dos traqueídeos $(3,54 \mu \mathrm{m})$ pode ser considerada espessa (entre 3 e $5 \mu \mathrm{m}$ ), de acordo com a classificação proposta por Manimekalai et al. (2002).

Na Tabela 3 é possível observar a comparação das características morfológicas dos traqueídeos da madeira de C. lanceolata com outras espécies de coníferas. Duan et al. (2016), avaliando clones da mesma espécie, oriundos de seis províncias da China, citaram que o comprimento dos traqueídeos variou entre 1,86 e 3,58 mm (média de 2,71 mm entre as províncias) e a largura entre 25,25 e 70,64 $\mu \mathrm{m}$ (média de 46,17 $\mu \mathrm{m}$ entre as províncias). Já Guo et al. (2018), estudando as dimensões dos traqueídeos da madeira de cerne e alburno de C. lanceolata, de diferentes idades (10, 20 e 30 anos), de duas províncias da China, observaram valores entre 1,40 e 3,77 mm para comprimento; 30,00 e $54,00 \mu \mathrm{m}$ para largura; 1,70 e 3,30 $\mu \mathrm{m}$ para espessura da parede celular.

Tabela 3. Comparação das dimensões dos traqueídeos da madeira de C. lanceolata aos 24 anos de idade, plantada em Campo Belo do Sul/SC, com outras espécies de coníferas.

\begin{tabular}{ccccccc}
\hline Espécie & $\begin{array}{c}\text { Idade } \\
\text { (anos) }\end{array}$ & $\mathbf{C}(\mathbf{m m})$ & $\mathbf{L}(\boldsymbol{\mu m})$ & $\mathbf{D L}(\boldsymbol{\mu m})$ & $\mathbf{E P}(\boldsymbol{\mu m})$ & Fonte \\
\hline C. lanceolata & 24 & 2,14 & 40,55 & 33,46 & 3,54 & Presente estudo \\
C. lanceolata & 20 & 2,28 & 44,00 & - & 2,40 & Guo et al. (2018) \\
C. japonica & 14 & 2,15 & 29,40 & 18,60 & 5,30 & Carneiro et al. (2009) \\
C. japonica & 22 & 2,21 & 30,10 & 19,20 & 5,50 & Pereira et al. (2003) \\
C. Iusitanica & 16 & 2,41 & 31,40 & 25,15 & 3,12 & Vivian et al. (2020) \\
P. taeda & 16 & 3,71 & 41,35 & - & 6,54 & Ampessan et al. (2015) \\
P. taeda & 21 & 3,50 & 40,55 & 27,71 & 6,41 & Vivian et al. (2015) \\
P. elliottii & 30 & 3,70 & 47,23 & - & 7,64 & Muñiz (1993) \\
P. sylvestris & 45 & 3,10 & 43,73 & 28,15 & 7,59 & Vivian et al. (2015) \\
\hline
\end{tabular}

Em que: $\mathrm{C}$ = comprimento; $\mathrm{L}=$ largura; $\mathrm{DL}$ = diâmetro do lúmen; $\mathrm{EP}$ = espessura da parede celular. 
O comprimento obtido para os traqueídeos da madeira de $C$. lanceolata no presente estudo é próximo ao citado na literatura para a mesma espécie, bem como para as madeiras de C. Iusitanica e C. japonica. Já quando comparado ao gênero Pinus, o comprimento dos traqueídeos da C. lanceolata são bem inferiores. Para a largura, os valores observados para a madeira de $C$. lanceolata são superiores aos observados para as madeiras de $C$. Iusitanica e C. japonica, mas são consoantes com o $P$. taeda.

Em relação ao diâmetro do lúmen dos traqueídeos, a C. lanceolata apresentou o maior valor quando comparada as outras espécies, isso porque seus elementos são relativamente largos, porém de parede celular mais fina em relação ao Pinus e $C$. japonica, mas similar a C. Iusitanica.

As dimensões e a morfologia dos traqueídeos podem ser informações importantes para determinar as potencialidades das madeiras visando à produção de polpa celulósica e papel, as quais podem ser utilizadas para o cálculo de indicadores anatômicos de qualidade, que auxiliam no entendimento do possível comportamento das células na formação do papel. $\mathrm{Na}$ Figura 5 observam-se as variações dos indicadores anatômicos e suas médias. Apenas o índice de enfeltramento (Figura 5D) apresentou diferença estatística significativa no sentido radial. A homogeneidade observada ao longo do raio para a fração parede, coeficiente de flexibilidade e índice de Runkel é interessante, pois indica que independentemente da porção utilizada do tronco, as propriedades do papel tenderão a se manterem as mesmas, o que é positivo no processo produtivo. Já o índice de enfeltramento, que aumenta significativamente no sentido medula-casca, indica que quanto mais velha a árvore melhor para este parâmetro, pois apresentará traqueídeos com maior capacidade de entrelaçamento entre si.
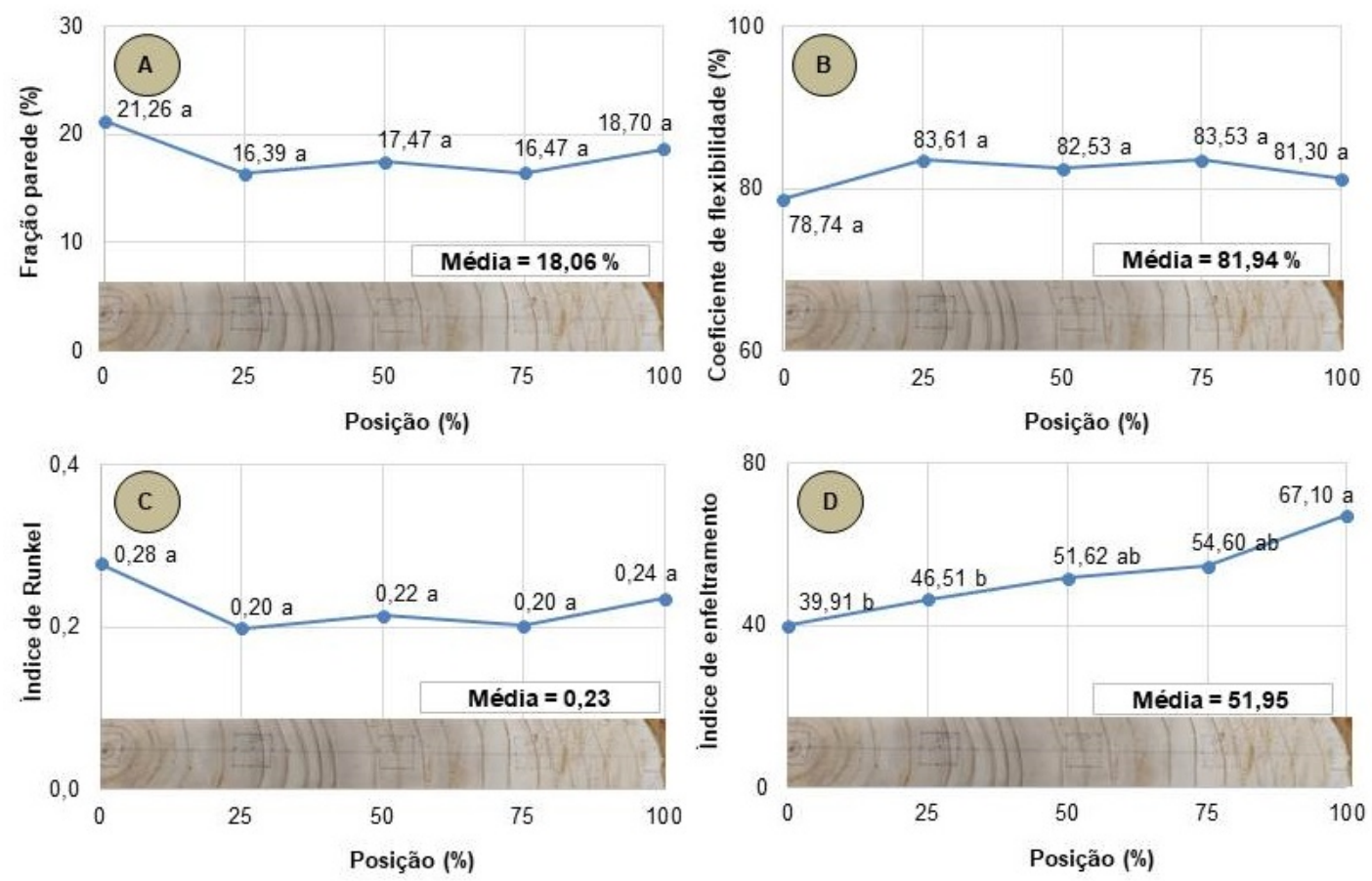

Figura 5. Indicadores anatômicos ao longo do raio da madeira de C. lanceolata aos 24 anos de idade, plantada em Campo Belo do Sul/SC. (A) Fração parede; (B) Coeficiente de flexibilidade; (C) Índice de Runkel; (D) Índice de enfeltramento. Média seguidas da mesma letra não diferem entre si pelos teste de Tukey.

Na Tabela 4 pode-se observar a comparação dos indicadores anatômicos da qualidade da madeira para fabricação de papel, entre a C. lanceolata com outras espécies de coníferas. De maneira geral, estas espécies de coníferas enquadram-se no segmento de "fibra longa", com papéis destinados a usos em embalagens ou finalidades que demandam maior resistência mecânica. 
Tabela 4. Comparação dos indicadores anatômicos de qualidade da madeira de C. lanceolata aos 24 anos de idade, plantada em Campo Belo do Sul/SC, com outras espécies de coníferas.

\begin{tabular}{ccccccc}
\hline Espécie & Idade (Anos) & FP (\%) & CF (\%) & IR & IE & Fonte \\
\hline C. lanceolata & 24 & 18,06 & 81,94 & 0,23 & 51,95 & Presente estudo \\
C. Iusitanica & 16 & 20,03 & 79,97 & 0,25 & 76,50 & Vivian et al. (2020) \\
P. taeda & 9 & 26,49 & 73,51 & 0,36 & 86,63 & Gomes (2009) \\
P. taeda & 16 & 29,98 & 70,02 & 0,47 & 93,44 & Ampessan et al. (2015) \\
P. taeda & 21 & 32,00 & 68,00 & 0,46 & 86,00 & Vivian et al. (2015) \\
P. sylvestris & 45 & 36,00 & 64,00 & 0,55 & 71,00 & Vivian et al. (2015)
\end{tabular}

Em que: $\mathrm{FP}$ = fração parede; $\mathrm{CF}$ = coeficiente de flexibilidade; IR = índice de Runkel; IE = índice de enfeltramento.

O valor de fração parede observado para a madeira de C. lanceolata foi inferior aos citados para as madeiras de C. Iusitanica, e para as espécies de Pinus. O valor obtido $(18,06 \%)$ permite enquadrá-la como muito delgada (<20\%), conforme a classificação de Klock (2013), que sugere que os traqueídeos apresentarão rigidez muito baixa. Shimoyama \& Wiecheteck (1993) mencionam que o ideal é que a fração parede seja menor que $40 \%$, pois as fibras/traqueídeos colapsariam com maior facilidade, proporcionando maior resistência ao papel (alta resistência à tração e estouro).

O coeficiente de flexibilidade observado para a madeira de $C$. lanceolata foi superior a todas as espécies utilizadas para comparação (Tabela 4). Tal coeficiente se relaciona com o grau de colapso das fibras/traqueídeos durante o processo da fabricação de papel, quanto mais alto tal indicador, melhor, pois maior a resistência à ruptura (Nisgoski et al., 2012). O valor obtido $(81,94 \%)$ indica que os traqueídeos da madeira de $C$. lanceolata apresentarão boa superfície de contato e união entre eles, que colapsarão com facilidade durante a formação do papel, com base na classificação mencionada por Nisgoski (2005), estando acima de 75\%. Costa (2011) afirma que quanto maior o coeficiente de flexibilidade, mais flexíveis serão os traqueídeos, resultando em maior resistência à tração e ao estouro do papel.

Outro indicador que demonstra o potencial da madeira de C. lanceolata para fabricação de papel é o índice de Runkel, que foi menor (nesse caso melhor, de acordo com a classificação de Runkel) quando comparada as outras espécies. Com base no valor observado $(0,23)$ para a madeira de $C$. lanceolata, é possível classificá-la como excelente para fabricação de papel, pois conforme escala criada por Runkel e citada por Tostes et al. (2013), o valor obtido se enquadra na categoria I $(<0,25)$, indicando que os traqueídeos são mais flexíveis e com alta capacidade de união entre os mesmos.

Por fim, o índice de enfeltramento obtido $(51,95)$ para a madeira de C. lanceolata foi inferior ao das demais espécies, porém está acima do mínimo citado por Nisgoski et al. (2012). A autora menciona que valores acima de 50 são interessantes pois conferem boas características ao papel em relação ao rasgo e arrebentamento.

De forma geral, com base nas propriedades físico-químicas e anatômicas da madeira de C. lanceolata, é possível salientar que ela apresenta pontos positivos e negativos, especialmente quando o intuito for utilizá-la para produção de celulose e papel. Atualmente ela competiria com as espécies do gênero Pinus, em especial com a espécie $P$. taeda, que é consagrada no segmento de "fibra longa", e que conta com um processo de melhoramento genético avançado. Talvez os principais pontos em que seja necessário mais atenção, são em relação à baixa densidade da madeira, seu alto teor de lignina e menor comprimento de traqueídeos, em relação ao Pinus. Entretanto vale a pena destacar seus ótimos indicadores anatômicos para produção de papel, que são superiores ao próprio Pinus.

Assim uma forma inicial, de curto e médio prazo, para aproveitar os aspectos positivos e contornar os negativos, seja a partir de um "mix" ou "blend" da madeira de C. lanceolata com a de Pinus. Já pensando no longo prazo, seria a implementação de programas de melhoramento genético e avaliação dos efeitos dos tratos silviculturais, visando adequar as características da madeira de $C$. lanceolata aos padrões ideais demandados pelo setor polpa e papel. 


\section{CONCLUSÕES}

Após a avaliação da madeira de C. lanceolata conclui-se que esta apresenta:

- densidade básica baixa ou leve;

- teores de cinzas e extrativos dentro da faixa normalmente observada para coníferas, porém valores elevados de lignina e baixo de holocelulose;

- traqueídeos largos, de comprimento moderadamente longo, de parede espessa, que variam de forma significativa no sentido radial (medula-casca);

- excelentes indicadores anatômicos para fabricação de papel, como índice de Runkel, coeficiente de flexibilidade e fração parede.

A partir da caracterização físico-química e anatômica da madeira de C. lanceolata, concluise que a mesma apresenta bom potencial para uso no Brasil, podendo ser uma alternativa viável para suprir a demanda de matéria-prima do setor industrial, especialmente para produção de celulose e papel. Além disso, avaliações de outros usos como construção civil e movelaria, merecem atenção, e devem ser mais explorados. Vale a pena destacar que a espécie não conta com programas de melhoramento genético, e ainda apresenta margem para melhoria de suas propriedades madeireiras.

\section{REFERÊNCIAS}

Almeida, C. C. F., Cunha, A. B., \& Rios, P. D. (2017). Avaliação da qualidade da colagem de topo da madeira de Cupressus lusitanica Mill. para a produção de painéis colados lateralmente. Scientia Forestalis, 45(113), 9-19. http://dx.doi.org/10.18671/scifor.v45n113.01.

Almeida, C. C. F., Rios, P. D., Cunha, A. B., Ampessan, C. G. M., \& Spanhol, A. (2016). Applicability evaluation of Cupressus lusitanica for pulp production. Maderas. Ciencia y Tecnología, 18(4), 651-662. http://dx.doi.org/10.4067/S0718-221X2016005000057.

Ampessan, C. G. M., Rios, P. D., Brand, M. A., Vieira, H. C., \& Kniess, D. D. C. (2015). Otimização do tempo de estocagem de cavacos de Pinus taeda e Pinus elliottii para a produção de celulose e papel. Scientia Forestalis, 43(108), 885-893. http://dx.doi.org/10.18671/scifor.v43n108.13.

Associação Brasileira de Normas Técnicas - ABNT. (2003). NBR-11941: madeira: determinação da densidade básica. Rio de Janeiro: ABNT.

Ballarin, A. W., \& Palma, H. A. L. (2003). Propriedades de resistência e rigidez da madeira juvenil e adulta de Pinus taeda L. Revista Árvore, 27(3), 371-380.

Barbosa, L. C. A., Maltha, C., \& Cruz, M. P. (2005). Composição química de extrativos lipofílicos e polares de madeira de Eucalyptus grandis. Revista Ciência \& Engenharia, 15(2), 13-20.

Barrichelo, L. E. G., \& Foelkel, C. E. B. (1983). Processo nítrico-acético para maceração de madeiras. Silvicultura, 28, 732-733.

Carneiro, M. E., Bittencourt, E., \& Muniz, G. I. B. (2009). Qualidade da madeira de Cryptomeria japonica D. DON. Floresta, 39(4), 913-920. http://dx.doi.org/10.5380/rf.v39i4.16328.

César, A., Bufalino, L., Mendes, L., Mesquita, R., Protásio, T., Mendes, R., \& Andrade, L. (2017). Transforming rice husk into a high-added value product: potential for particleboard production. Ciência Florestal, 27(1), 303-313. http://dx.doi.org/10.5902/1980509826468.

Chen, M., Wang, C., Fei, B., Ma, X., Zhang, B., Zhang, S., \& Huang, A. (2017). Biological degradation of Chinese fir with Trametes versicolor (L.) lloyd. Materials, 10(7), 834. http://dx.doi.org/10.3390/ma10070834.

Costa, J. A. (2011). Qualidade da madeira de Eucalyptus urograndis, plantado no Distrito Federal, para produção de celulose Kraft (Dissertação de mestrado). Universidade de Brasília, Brasília.

Dias, O. A., \& Simonelli, G. (2013). Qualidade da madeira para a produção de celulose e papel. Enciclopédia Biosfera, 9(17), 3632-3646.

Dobner Júnior, M., Huss, J., \& Tomazello Filho, M. (2018). Wood density of loblolly pine trees as affected by crown thinning and harvest age in southern Brazil. Wood Science and Technology, 52(2), 465-485. http://dx.doi.org/10.1007/s00226-017-0983-9.

Dobner Júnior, M., Trazzi, P. A., Machado, A. A., \& Higa, A. R. (2017). Aspectos dendrométricos e silviculturais de um povoamento de Cunninghamia lanceolata no sul do Brasil. Floresta, 47(1), 1-9. http://dx.doi.org/10.5380/rf.v47i1.43562. 
Duan, H., Cao, S., Zheng, H., Hu, D., Lin, J., Lin, H., Hu, R., Sun, Y., \& Li, Y. (2016). Variation in the growth traits and wood properties of chinese fir from six provinces of Southern China. Forests, 7(12), 192. http://dx.doi.org/10.3390/f7090192.

Empresa Brasileira de Pesquisa Agropecuária - EMBRAPA. (1988). Zoneamento ecológico para plantios florestais no estado de Santa Catarina. Curitiba: EMBRAPA.

Fantuzzi Neto, H. (2012). Qualidade da madeira de eucalipto para produção de celulose kraft (Tese de doutorado). Universidade Federal de Viçosa, Viçosa.

Fonte, A. P. N., Trianoski, R., Iwakiri, S., \& Anjos, R. A. M. (2017). Propriedades físicas e químicas da madeira de cerne e alburno de Cryptomeria japonica. Revista de Ciências Agroveterinárias, 16(3), 277. 285. http://dx.doi.org/10.5965/223811711632017277.

Fredo, A., Foelkel, C. E. B., Frizzo, S. M. B., \& Silva, M. C. M. (1999). Elementos minerais em madeiras de eucaliptos e acácia negra e sua influência na indústria de celulose kraft branqueada. Ciência Florestal, 9(1), 193-209. http://dx.doi.org/10.5902/19805098376.

Fung, L. E. (1993). Wood properties of new zealand-grown Cunninghamia lanceolata. New Zealand Journal of Forestry Science, 23(3), 324-338.

Gomes, F. A. (2009). Avaliação dos processos Kraft convencional e Lo-Solids para madeira de Pinus taeda (Dissertação de mestrado). Escola Superior de Agricultura “Luiz de Queiroz”, Universidade de São Paulo, Piracicaba.

Guo, J., Guo, X., Xiao, F., Xiong, C., \& Yin, Y. (2018). Influences of provenance and rotation age on heartwood ratio, stem diameter and radial variation in tracheid dimension of Cunninghamia lanceolata. European Journal of Wood and Wood Products, 76(2), 669-677. http://dx.doi.org/10.1007/s00107-017-1200-0.

International Association of Wood Anatomists - IAWA. (1989). List of microscopic features for hardwood identification. IAWA Bulletin, 10(3), 219-332.

Klock, U. (2013). Polpa e papel: propriedades do papel (53 p.). Curitiba: Setor de Ciências Agrárias, Departamento de Engenharia e Tecnologia Florestal, Universidade Federal do Paraná. Recuperado em 20 de julho de 2020, de http://www.madeira.ufpr.br/disciplinasklock/polpaepapel/papelpropriedades2013.pdf

Klock, U., \& Andrade, A. S. (2013). Química da madeira (4. ed., 87 p.). Curitiba: UFPR.

Li, Y., Deng, X., Zhang, Y., Huang, Y., Wang, C., Xiang, W., Xiao, F., \& Wei, X. (2019). Chemical characteristics of heartwood and sapwood of red-heart Chinese Fir (Cunninghamia lanceolata). Forest Products Journal, 69(2), 103-109. http://dx.doi.org/10.13073/FPJ-D-18-00042.

Manimekalai, V., Pavichandran, P., \& Balasubramanian, A. (2002). Fibres of Sorghum bicolor (L.) Moench and their potential use in paper and board making. Phitomorphology, 52(1), 55-59.

Mattos, B. D., Gatto, D. A., Stangerlin, D. M., Calegari, L., Melo, R. R., \& Santini, E. J. (2011). Variação axial da densidade básica da madeira de três espécies de gimnospermas. Agrária, 6(1), 121-126. http://dx.doi.org/10.5039/agraria.v6i1a1080.

Melo, R. R., Silvestre, R., Oliveira, T. M., \& Pedrosa, T. D. (2013). Variação radial e longitudinal da densidade básica da madeira de Pinus elliottii Engelm. com diferentes idades. Ciência da Madeira, $4(1), 83-92$.

Metcalfe, C. R., \& Chalk, L. (1983). Anatomy of the dicotyledons: wood structure and conclusion of the general introduction (2nd ed., 308 p.) Oxford: Clarendon Press.

Minghe, L., \& Ritchie, G. A. (1999). Eight hundred years of clonal forestry in China: I. traditional afforestation with Chinese fir (Cunninghamia lanceolata (Lamb.) Hook.). New Forests, 18(2), 131-142. http://dx.doi.org/10.1023/A:1006558900234.

Muñiz, G. I. B. (1993). Caracterização e desenvolvimento de modelos para estimar as propriedades e o comportamento na secagem da madeira de Pinus elliottii Engelm. e Pinus taeda L. (Tese de doutorado). Universidade Federal do Paraná, Curitiba.

Nisgoski, S. (2005). Espectroscopia no infravermelho próximo no estudo de características da madeira e papel de Pinus taeda L. (Tese de doutorado). Universidade Federal do Paraná, Curitiba.

Nisgoski, S., Muñiz, G. I. B., Trianoski, R., Matos, J. L. M., \& Venson, I. (2012). Características anatômicas da madeira e índices de resistência do papel de Schizolobium parahyba (Vell.) Blake proveniente de plantio experimental. Scientia Forestalis, 40(94), 203-211.

Palermo, G. P., Latorraca, J. V. F., Severo, E. T. D., Rezende, M. A., \& Abreu, H. S. (2004). Determinação da densidade da madeira de Pinus elliottii Engelm, através de atenuação de radiação gama comparada a métodos tradicionais. Floresta e Ambiente, 11(1), 1-6. 
Pereira, J. C. D., Higa, R. C. V., \& Shimizu, J. Y. (2003). Propriedades da madeira do cedrinho japonês (Comunicado Técnico, No. 88, 4 p.). Colombo: Embrapa Florestas. Recuperado em 24 de julho de 2020, de https://ainfo.cnptia.embrapa.br/digital/bitstream/CNPF-2009-09/35544/1/Com_tec88.pdf

Segura, T. E. S. (2015). Avaliação das madeiras de Corymbia citriodora, Corymbia torelliana e seus híbridos visando à produção de celulose kraft branqueada (Tese de doutorado). Escola Superior de Agricultura "Luiz de Queiroz", Universidade de São Paulo, Piracicaba.

Shimoyama, V. R. S., \& Wiecheteck, M. S. S. (1993). Características da madeira e da pasta termomecânica de Pinus patula var. tecunumanii para produção de papel imprensa. IPEF, 9(27), 63-80.

Technical Association of Pulp and Paper Industry - TAPPI. (1997). TAPPI T204 cm-97: solvent extractives of wood and pulp. Atlanta: TAPPI Press.

Technical Association of Pulp and Paper Industry - TAPPI. (2002). TAPPI T211 om-02: ash in wood, pulp, paper and paperboard: combustion at $525^{\circ} \mathrm{C}$. Atlanta: TAPPI Press.

Tostes, L. C. L., Machado, S. R., Carim, M. V., \& Guimarães, J. R. S. (2013). Morfometria das fibras do sistema radicular de Philodendron bipinnatifidum Schott (Família Araceae; subgênero Meconostigma). Biota Amazônia, 3(3), 15-22. http://dx.doi.org/10.18561/21795746/biotaamazonia.v3n3p15-22.

Trianoski, R., Iwakiri, S., Matos, J. L. M., \& Chies, D. (2013a). Utilização da madeira de Cryptomeria japonica para produção de painéis aglomerados. Scientia Forestalis, 41(97), 57-64.

Trianoski, R., Matos, J. L. M., Iwakiri, S., \& Prata, J. G. (2013b). Avaliação da estabilidade dimensional de espécies de pinus tropicais. Floresta e Ambiente, 20(3), 398-406. http://dx.doi.org/10.4322/floram.2012.071.

Vivian, M. A., Corrêa, R., Modes, K. S., Caetano, A. P., Pedrazzi, C., \& Dobner Júnior, M. (2020). Caracterização tecnológica da madeira de Cupressus lusitanica visando à produção de polpa celulósica. Pesquisa Florestal Brasileira, 40(e201901894), 1-9. http://dx.doi.org/10.4336/2020.pfb.40e201901894.

Vivian, M. A., Segura, T. E. S., Bonfatti Júnior, E. A., Sarto, C., Schmidt, F., Silva Júnior, F. G., Gabov, K., \& Fardim, P. (2015). Qualidade das madeiras de Pinus taeda e Pinus sylvestris para a produção de polpa celulósica kraft. Scientia Forestalis, 48(105), 183-191.

Wang, J., Li, J., Li, S., Freitag, C., \& Morrell, J. J. (2011). Antifungal activities of Cunninghamia lanceolata heartwood extractives. BioResources, 6(1), 606-614. http://dx.doi.org/10.15376/biores.6.1.606-614.

Wen, H., Deng, X., Zhang, Y., Wei, X., Wang, G., Zhou, B., Xiang, W., \& Zhu, N. (2018). Cunninghamia lanceolata variant with red-heart wood: a mini-review. Dendrobiology, 79, 156-167. http://dx.doi.org/10.12657/denbio.079.014.

Xin, H. L., Zhai, X. F., Zheng, X., Zhang, L., Wang, Y. L., \& Wang, Z. (2012). Anti-inflammatory and analgesic activity of total flavone of Cunninghamia lanceolata. Molecules, 17(8), 8842-8850. http://dx.doi.org/10.3390/molecules17088842.

Zheng, H., Hu, D., Wang, R., Wei, R., \& Yan, S. (2015). Assessing 62 Chinese fir (Cunninghamia lanceolata) breeding parents in a 12-year grafted clone test. Forests, 6(12), 3799-3808. http://dx.doi.org/10.3390/f6103799.

Contribuição dos Autores: MAV: Curadoria de Dados, Metodologia, Escrita - Primeira Redação, Supervisão, Escrita - Revisão e Edição; AMOS: Investigação, Metodologia; KSM: Conceituação, Escrita - Revisão e Edição; MDJ: Escrita - Revisão e Edição, Validação; FGSJ: Metodologia, Escrita - Revisão e Edição. 